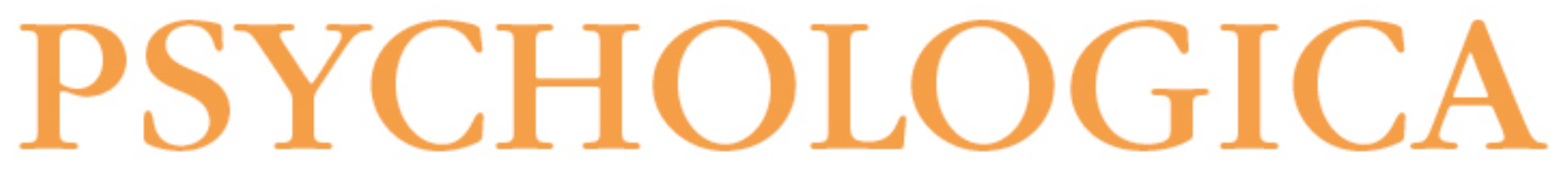

Uma analise critica da revolucao cognitiva: implicacoes epistemologicas

Autor(es): $\quad$ Lopes, Ederaldo José; Lopes, Renata Ferrarez Fernandes

Publicado por: Imprensa da Universidade de Coimbra

URL

persistente: URI:http://hdl.handle.net/10316.2/5434

DOI: $\quad$ DOI:http://dx.doi.org/10.14195/1647-8606_50_1

Accessed : $\quad$ 26-Apr-2023 08:59:47

A navegação consulta e descarregamento dos títulos inseridos nas Bibliotecas Digitais UC Digitalis, UC Pombalina e UC Impactum, pressupõem a aceitação plena e sem reservas dos Termos e Condições de Uso destas Bibliotecas Digitais, disponíveis em https://digitalis.uc.pt/pt-pt/termos.

Conforme exposto nos referidos Termos e Condições de Uso, o descarregamento de títulos de acesso restrito requer uma licença válida de autorização devendo o utilizador aceder ao(s) documento(s) a partir de um endereço de IP da instituição detentora da supramencionada licença.

Ao utilizador é apenas permitido o descarregamento para uso pessoal, pelo que o emprego do(s) título(s) descarregado(s) para outro fim, designadamente comercial, carece de autorização do respetivo autor ou editor da obra.

Na medida em que todas as obras da UC Digitalis se encontram protegidas pelo Código do Direito de Autor e Direitos Conexos e demais legislação aplicável, toda a cópia, parcial ou total, deste documento, nos casos em que é legalmente admitida, deverá conter ou fazer-se acompanhar por este aviso. 


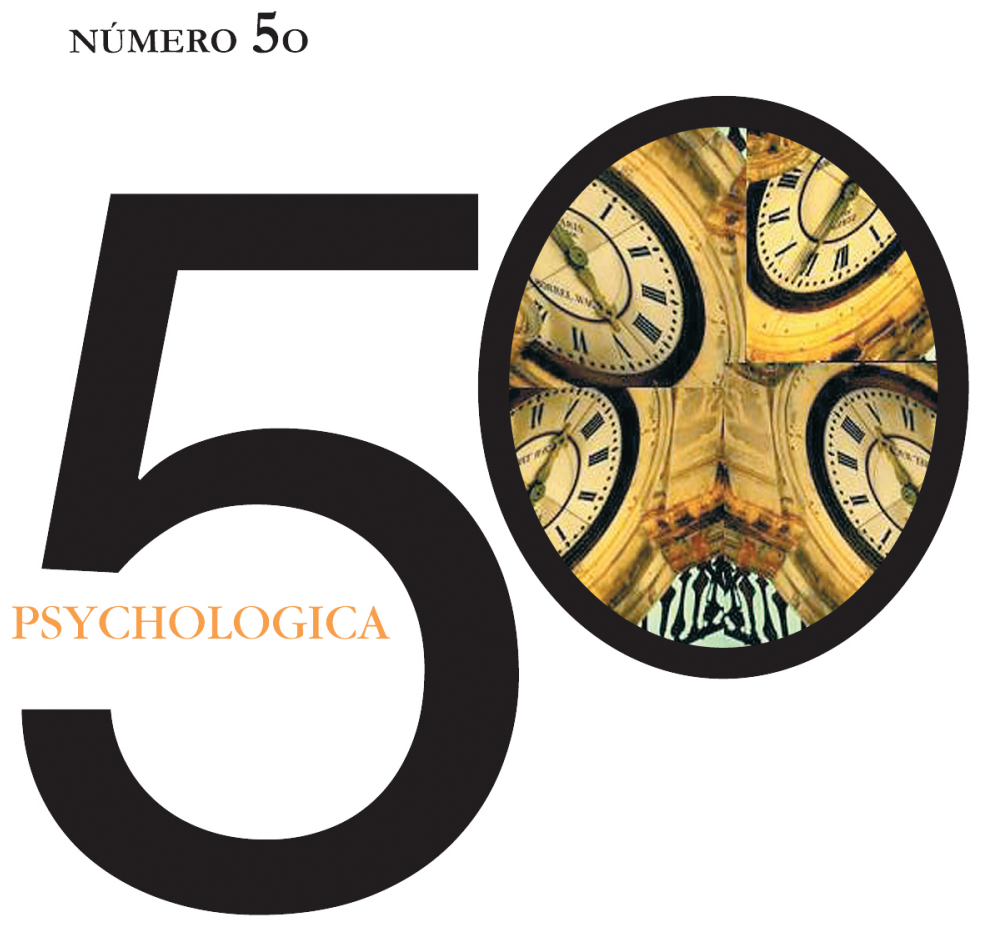

IMPRENSA DA UNIVERSIDADE DE COIMBRA

FACULDADE DE PSICOLOGIA E DE CIÊNCIAS DA EDUCAÇÃO DA UNIVERSIDADE DE COIMBRA 


\title{
Uma Análise Crítica da Revolução Cognitiva: Implicações Epistemológicas
}

\author{
Ederaldo José Lopes` \& Renata Ferrarez Fernandes Lopes²
}

Este artigo apresenta um histórico da revolução cognitiva em psicologia, uma discussão crítica sobre ela e as implicações epistemológicas para o status científico da psicologia. Surgida na década de 50 e tendo o paradigma do processamento de informação como base, a psicologia cognitiva foi difundida como uma revolução científica, tal como proposta por T. Kuhn. Essa proposta se mostrou falsa, colocando dúvidas sobre a cientificidade da psicologia. Propõe-se o abandono da filosofia de Kuhn e a adoção do naturalismo normativo como uma saída frutífera para as psicologias.

PALAVRAS-CHAVE: Psicologia Cognitiva; Paradigma; Revolução Científica; Revolução Cognitiva; Naturalismo Normativo.

\section{Uma Análise Crítica da Revolução Cognitiva: Implicações Epistemológicas.}

\section{A retomada da mente: Um pouco de história}

Desde os seus primórdios enquanto uma ciência, a psicologia se constituiu numa série de escolas ou sistemas (Heidbreder, 1933/1981). Esses sistemas têm a importante missão de estimular o pensamento e servir de meios de aquisição dos fatos científicos. A psicologia cognitiva moderna (processamento da informação ou $\mathrm{Pl}$ ) pode ser considerada como um desses sistemas. Do mesmo modo que seu antecessor (o behaviorismo), lançou-se às pesquisas tomando por base uma metodologia de pesquisa essencialmente calcada na experimentação. Essa psicologia retoma o mentalismo na psicologia, tendo herdado as questões postas pela filosofia (O que é o pensamento? A linguagem influencia o pensamento? As memórias e as percepções são precisas?). A psicologia cognitiva já pode ser

\footnotetext{
1 Instituto de Psicologia, Universidade Federal de Uberlândia

Presidente da Sociedade Brasileira de Psicologia

2 Instituto de Psicologia, Universidade Federal de Uberlândia
} 
encontrada em Aristóteles, podendo ser considerada, sob muitos aspectos, uma espécie de filosofia empírica (Hatfield, 2002; Levitin, 2002).

A história da psicologia cognitiva se confunde, de início, com a história da psicologia (Anderson, 1995). Antes mesmo de Wundt, os estudos de Donders (1868/1969), baseados nas descobertas da velocidade da condução nervosa na rã feitas por Helmholtz (1852, cit. in De Jaager, 1970), são um marco importante da cronometria mental (Galera \& Lopes, 1995). A tese de Ebbinghaus (1885/1964) foi um trabalho importante em que se estudaram os recursos associativos na memória de curto prazo, tendo sido uma técnica usada em vários trabalhos de investigação da memória no paradigma experimental da aprendizagem serial (Kausler, 1974), bem como uma evidência de que o método da repetição (recitação interna de uma informação) é um elemento fundamental do processo de memorização (Sternberg, 2007). Enquanto Wundt fazia uma psicologia cognitiva mais sensorial e fisiológica, William James (1890/1950) mostrou uma preocupação maior - mesmo que de uma forma especulativa - com os aspectos cognitivos mais próximos do que diz Neisser (1967) ser o objeto de estudo dessa área (Kendler, 1987; Kessel \& Bevan, 1985). É o caso da psicologia da memória: Em seu Princípios, James estabeleceu uma importante distinção entre memória primária e secundária que, na década de 6o, deu origem ao que conhecemos como memória de curto prazo e memória de longo prazo (Atkinson \& Shiffrin, 1968) Na mesma obra, James forneceu outros conceitos importantes, dentre os quais o conceito de atenção merece destaque (La Berge, 1990; Lopes \& Galera, 1998; Sternberg, 2007).

Um outro marco importante foi a pesquisa de Tolman (1932) sobre a aprendizagem em labirinto com camundongos, sugerindo que os animais formavam um mapa cognitivo do labirinto, facilitando a busca de alimento. De certa forma, isso foi um prenúncio da idéia-chave da psicologia cognitiva, a idéia de representação mental (Lachman, Lachman, \& Butterfield, 1979; Mandler, 1985).

Foi na década de 50 que o movimento cognitivista floresceu de fato. Vários eventos contribuíram substancialmente para esse florescimento: a teoria da informação (Shannon \& Weaver, 1949); o estudo dos fatores humanos e as relações homem-máquina (engineering psychology) após a II Guerra Mundial (Broadbent, 1958; Lachman et al., 1979); o artigo de George Miller (1956) sobre o número mágico 7 contribuiu para os primeiros entendimentos sobre as limitações da memória de curto prazo; as pesquisas do lingüista Noam Chomsky (1959) e a revisão de "O Comportamento Verbal" de Skinner (1957/1978) incendiaram o debate a respeito da natureza da linguagem; o advento do computador, que serviu como modelo para o estudo dos processos mentais (Crane, 1995; Eysenck \& Keane, 1994; French \& Colman, 1995; Teixeira, 1998, 2000; Thagard, 1998). 
Finalmente, no campo da epistemologia, os ataques de filósofos como K. Popper (1959/1999) à idéia de objetividade científica e indução, assim como a idéia de revolução científica (Kuhn, 1962/1978; Masterman, 1979; Popper, 1979) estabeleceram a possibilidade de se pensar que um novo "paradigma" havia surgido na psicologia.

\section{A revolução cognitiva: fato ou ficção?}

A análise de vários textos, incluindo livros ou artigos, mostra que o uso da expressão revolução cognitiva foi inflacionada na psicologia. Na visão kuhniana, o surgimento de um novo paradigma só poderia ocorrer após um período de crise de um paradigma anterior. Para os que advogam pela causa da revolução, a década de 50 viu a queda do behaviorismo e o nascimento da psicologia cognitiva. Com efeito, ter-se-ia estabelecido uma revolução, dando surgimento ao "paradigma" do processamento de informação na psicologia? Vamos aos fatos:

Lachman et al. (1979) identificaram a metáfora do processamento de informação como um paradigma Kuhniano para a psicologia cognitiva:

When a sufficiently large number of scientists in a field agree to a considerable extent on how such questions are to be solved, they are said to share a "paradigm". Information-processing psychology is one of paradigm for studying cognitive psychology, and it happens that in recent years it has become the dominant paradigm in the study of adult cognitive processes (p. 6).

Os autores continuam a descrever de forma mais aprofundada as noções de paradigma e revolução científica e a tônica do livro era fortalecer a ideia de que estabeleceu-se um paradigma e, mais do que isso, era necessário tornar este conhecimento ao público, tal como ocorreu com as ideias freudianas e comportamentais. Para isso dedicaram mais três capítulos do livro para discorrer sobre o processamento de informação.

Para Kessel e Bevan (1985),

As described here, cognitive psychology, whether on its own or as one segment of cognitive science, is generally equated with the information-processing metaphor or model, and therefore taken to represent or incorporate a "paradigm shift" (p. 263).

Ao apresentar a história da psicologia cognitiva, Kendler (1987) afirmou:

Thus, the stimulus-response psychology of Hull and his disciples, which had its roots in the theoretical analysis of animal learning, was gradually, expanding to the cognitive processes of humans. Why did psychology have to experience a 
cognitive revolution instead of gradual evolutionary development of stimulus-response learning theories to encompass human cognition? (p. 374).

Vários discursos de teóricos cognitivos vão no mesmo sentido, ou seja, de fazer uma demarcação da psicologia cognitiva como um movimento de retomada da mente na psicologia, em oposição ao behaviorismo, retomada esta feita em clima revolucionário. Até mesmo Skinner (1991) afirmou:

A palavra de ordem da revolução cognitiva é: “A mente está de volta!". Nasce uma "grande nova ciência da mente". O comportamentalismo quase destruiu nosso interesse pelo assunto, mas foi derrotado, e devemos retomar o fio da meada onde filósofos e os primeiros psicólogos pararam. (p. 38)

Além da famosa obra de Lachman et al. (1979), outros manuais de psicologia cognitiva também apresentam as idéias de Kuhn como sustentáculos para o aparecimento da nova abordagem revolucionária na Psicologia. Num desses manuais (Eysenck \& Keane 1994), já no primeiro capítulo, apresentam como um dos tópicos importantes para o aparecimento da abordagem cognitiva as alterações nas visões de ciência, uma das quais é a noção de paradigma de Kuhn. A visão de que a psicologia cognitiva foi um movimento revolucionário foi apresentada por Gardner (1995), em cuja obra, a começar do próprio título, já está expressa a revolução. A mesma tônica foi seguida por Baars (1996) e por aqueles que já falam no futuro da revolução (Johnson \& Erneling, 1997; Solso, 1999). Para Miller (2003), um dos "fundadores" do movimento cognitivista, a revolução cognitiva foi uma contra-revolução, no sentido de que foi uma reação a uma primeira revolução na psicologia, a revolução comportamental.

Algumas obras, todavia, abrandam o sentido revolucionário do movimento cognitivo, como, por exemplo, Matlin (2004), que fala de um entusiasmo crescente pela abordagem cognitiva e Goldstein (2005), que afirma a existência de um movimento que objetivou o ressurgimento do estudo da mente, porém não enfatizando que esse movimento tenha representado uma revolução.

Os pontos convergentes quanto à existência de uma revolução cognitiva mostram formas irrefletidas da noção de paradigma e de revolução científica de Kuhn (1962/1978). Essa irreflexão pode ser constatada pelo mau uso dos conceitos kuhnianos, revelando talvez não só uma imprecisão no uso dos termos, mas um desejo inveterado de justificar a cientificidade da psicologia. A obra de Kuhn parece ter sido o grito de liberdade de muitos psicólogos num momento em que, inclusive, as revoluções no terreno político avançavam. No Brasil, é duvidável a aplicação da noção de revolução ao golpe militar de 1964 (Castelo Branco, 2007). No campo das ciências, pode-se perceber o mesmo fenômeno. Um balanço entre dados empíricos, a partir de estudos bibliométricos (Robins, Gosling, \& Craik, 2000; Spear, 2007), e a contextualização das ideias de Kuhn podem ajudar a esclarecer esses equívocos. 
A pergunta, então, é a seguinte: por que muitos psicólogos afrouxaram as noções de Kuhn para uso indiscriminado?

Não há dúvida do alcance e popularidade despertados pela Estrutura das Revoluções cientificas (Kuhn, 1962/1978). Entre outras novidades, ele introduz a noção de que a ciência não é vista pelo acúmulo de descobertas, mas trata-se de um processo histórico; além disso, a ciência é um processo sociológico e subjetivo, colocando a noção de irracionalidade na arena da atividade científica, o que Ihe rendeu severas críticas. Ademais, a ciência progride via estágios de desenvolvimento que vão de um estágio pré-paradigmático, passando pelo estágio paradigmático, crise e revolução, dando origem, então, a um novo paradigma. Resumidamente, um paradigma é uma maneira aceita de resolução de problemas, além de um compartilhamento de valores, crenças, etc., pelos membros de uma comunidade científica. Um paradigma que entra em anomalias passa a sofrer pressões pelas novas idéias produzidas, o período dito revolucionário. Nesse período um paradigma substitui outro, não somente para dar conta das anomalias, mas porque houve produção de novas visões de mundo, novas crenças, surgindo inclusive novos problemas não colocados pelo paradigma anterior. Num exegético livro, Carone (2003) fez um levantamento dos conceitos kuhnianos e dos seus usos e abusos no meio psicológico, através da análise de publicações. Ela propõe uma revisão crítica do uso inflacionado das idéias de Kuhn na psicologia, ao mesmo tempo em que oferece respostas convincentes sobre as questões por nós colocadas anteriormente. Para Carone,

Atuando no contra-ataque às filosofias das ciências positivistas e neopositivistas, que sempre a consideraram como um conhecimento pré-científico, a Psicologia tentou encontrar na ideia de paradigma uma expressão feliz de sua cientificidade, transformando em "riqueza de conhecimentos" a multiplicidade de suas teorias e das práticas clínicas divergentes (p. 13).

Carone (2003) conclui seu trabalho apontando algumas razões pelas quais Kuhn vem sendo usado (ou mal usado) na psicologia: o receio de que a psicologia caia no limbo da pseudociência; o uso do conceito de paradigma para demarcar territórios dentro da própria psicologia, o que, a meu ver, é mais um elemento que revela a desintegração da psicologia; o anúncio repetido de revoluções parece servir ao propósito de enterrar os "rivais"; o conceito de paradigma parece dar uma certa legitimação à "torre de Babel das psicoterapias", em nome de um "ecletismo multiparadigmático" (Carone, 2003, p. 112); o uso do conceito de paradigma para referendar a Psicologia como uma ciência "pós-moderna."

Em trabalho anterior, Carone (1998) já chamava atenção para a dificuldade de se falar em paradigmas na psicologia e nas ciências sociais. Um dos problemas apontados era a falta de universalidade de acordo quanto às realizações científicas, 
problemas e soluções encontradas no seio de uma comunidade científica, algo que pode ser visto nas ciências caracterizadas pela existência de diversas escolas de pensamento. É o caso da psicologia que, na conhecida história escrita por Heidbreder (1933/1981), foi intitulada Seven psychologies. Quantas psicologias temos hoje? A questão da fragmentação do conhecimento psicológico é um fato que não pode ser negado. Como existir universalidade de acordo sobre um tipo de conhecimento caracterizado pela existência de inúmeros métodos, modelos e teorias?

Cronbach (1957) apresenta-nos um quadro dessa diversidade:

The scene resembles that of a circus, but a circus grander and more bustling than any Barnum ever envisioned - a veritable week-long diet of excitement and pink lemonade. (p. 671).

Nessa pletora de sistemas e teorias, o caráter revolucionário dado tanto ao behaviorismo quanto ao cognitivismo foi tratado como "as revoluções míticas da psicologia americana" (Leahey, 1992). Além disso, os sinais de desagregação permanecem vívidos, de modo que uma possível unificação do conhecimento psicológico parece improvável (Driver-Linn, 2003; Hunt, 2005; Spear, 2007). Spear (2007) argumenta que só faz sentido falar em revoluções na psicologia se ela fosse uma disciplina unificada. Spear menciona que o ceticismo e a incerteza quanto à revolução cognitiva vêm gerando uma série de análises baseadas em dados de publicação nos periódicos mais importantes da área de psicologia. Os dados de Spear mostram que a psicologia cognitiva vem se desenvolvendo em importância, porém sem dominância, a atenção dada à neurociência é similar àquela dada à psicologia cognitiva, enquanto a neurociência cognitiva está em processo de emergência (Brehm, 2008; Lopes, Lopes, \& Rodrigues, 2000; Posner \& Levitin, 1999; Solso, 1999; Thompson, 1999). Friman, Allen, Kerwin e Larzelere (1993) obtiveram resultados que mostram que a psicologia cognitiva tornou-se mais importante nas décadas recentes, porém isso não sustenta a ideia de que houve uma revolução. Se assim fosse, não haveria a co-existência do behaviorismo e da psicanálise.

A alegação de que o surgimento da psicologia cognitiva não representou uma mudança paradigmática no curso da ciência psicológica recebeu, recentemente, um forte apelo, sustentado por diversas visões dos processos de desenvolvimento da ciência, bem como pela voz de cientistas cognitivos de renome. Desse modo, numa análise também bastante pormenorizada da revolução cognitiva feita por O'Donohue, Ferguson e Naugle (2003), eles mostraram que essa revolução foi mais um movimento sociológico e retórico do que propriamente uma revolução kuhniana. Assim, à questão teria havido uma revolução cognitiva na psicologia, a exemplo do que ocorreu nas ciências fisicas?, we argue that the so-colled cognitive revolution is best characterized as a socio-rethorical phenomenon (O'Donohue et al., 
2003, p. 85). Entretanto, os autores não discordam da popularidade da psicologia cognitiva (ver também Hassebrock, 1990):

There is no denying the fact that cognitive psychology has grown in popularity at a faster rate over the last three decades than behavioral psychology has. A recent citation analysis lends some support to this claim (Friman, Allen, Kerwin, \& Larzelere, 1993). However, unlike a bona fide scientific revolution, this shift in emphasis is best characterized as a sociological phenomenon - a change in allegiance, that, interestingly, may be due in part to the claim (which has immense rhetorical value) that a scientific revolution has indeed taken place (p. 86).

A argumentação desses autores foi fundamentada a partir de cinco visões de ciência de eminentes filósofos da ciência contemporâneos: Popper, Kuhn, Lakatos, Laudan e Gross. Essas visões foram complementadas por dados obtidos a partir da opinião emitida por eminentes participantes da revolução cognitiva.

A partir da visão de Popper (1959/1999), as teorias comportamentais não foram falseadas pelas teorias cognitivas. Além disso, não há nada que mostre que a teoria cognitiva tenha uma quantidade maior de informação empírica, seja mais falseável e tenha maior poder explanatório ou preditivo que a teoria comportamental.

A segunda visão exposta pelos autores é a filosofia da ciência de Kuhn, já abordada anteriormente. Do mesmo modo que outros autores, a conclusão de O'Donohue et al. (2003) é que não há por que se falar em revolução cognitiva sem falar em paradigmas e mesmo que houvesse os paradigmas comportamental e cognitivista, o paradigma comportamental teria que ter entrado num mar de anomalias e ser suplantado pelo cognitivismo, o que não houve, com efeito.

A terceira visão é a de Lakatos (1981), que substituiu a concepção Kuhniana de paradigma pela noção de programa de pesquisa. Um programa de pesquisa se diz progressivo quando seus desenvolvimentos teóricos antecipam seus desenvolvimentos empíricos, mantendo a capacidade de predizer novos fatos com relativo sucesso. Entretanto, um programa de pesquisa pode fracassar na tarefa de predizer novos fatos e, neste caso, nós falamos em programa estagnado ou degenerado. De acordo com Lakatos (1981), um programa de pesquisa progressivo pode competir com um programa que está em processo de degeneração, podendo mesmo suplantá-lo, resultando daí uma revolução científica. Assim, a pergunta que cabe é: teria havido essa substituição do behaviorismo pelo cognitivismo, o que equivale dizer que o programa cognitivista (progressivo) substituiu o programa behaviorista (degenerado)? Para haver uma revolução cognitiva, o programa behaviorista teria que ter parado de proporcionar novas predições e sucessos empíricos (dados para suporte teórico), de modo que as anomalias deveriam ser 
encontradas via manobras ad hoc em vez de se introduzirem novas teorias. Nada disso pode ser constatado.

A quarta visão é a de Laudan (1977): Laudan criou a expressão tradição de pesquisa. Resumindo, uma tradição de pesquisa é uma família de teorias que compartilham uma ontologia e uma metodologia comuns. Para ele o objetivo de uma teoria é apresentar soluções que busquem resolver as ambiguidades entre as teorias, com o propósito de reduzir as irregularidades. Há três problemas empíricos: os resolvidos, os não-resolvidos e os anômalos. Dos três, o mais importante para o progresso científico e a ciência revolucionária é a noção de problema anômalo. Quando uma tradição de pesquisa concorrente resolve um problema até então não-resolvido, o problema não-resolvido torna-se um problema anômalo, colocando em dúvida a tradição fracassada. O’Donohue et al. (2003) concluem, à luz dos dados disponíveis, pela implausibilidade em se aceitar que houve uma revolução cognitiva de acordo com Laudan. Não há consenso dentro da nova tradição de que o movimento cognitivo foi capaz de resolver mais problemas empíricos do que o behaviorismo, nem de ter resolvido mais dados anômalos, assim como não se mostrou capaz de resolver um número maior de problemas conceituais.

Finalmente, os autores apresentam a perspectiva retórica Grossiana: Em ciência, como em muitas outras áreas (direito, educação, artes), a "arte da persuasão" é um expediente utilizado para o convencimento do público, e essa arte tem sido estudada pela retórica. A retórica também pode ser usada para convencer os cientistas a estudar determinados problemas, a publicar artigos, buscar financiamentos em agências de pesquisas. A significância dessas coisas depende da arte do convencimento, depende de julgamentos e argumentação. No caso da filosofia da ciência, o debate sobre qual é o método apropriado para se investigar um determinado assunto é um caso de persuasão, mais que de lógica. A propósito, essas questões foram levadas ao extremo quando Feyrabend (1975) escreveu seu famoso "Contra o Método", onde ele argumenta convincentemente que as metodologias da ciência não forneceram regras claras que guiassem as atividades dos cientistas. Então, a única regra válida, generalizada, é que "qualquer coisa serve". Mais ainda, segundo ele, a ciência progride através da estratégia do tipo "quem berra mais alto", em que a publicidade, a persuasão, a aparência valem mais do que a qualidade da pesquisa. A palavra retórica freqüentemente tem uma conotação negativa. Neste caso, quer dizer que o uso da persuasão pode ser feita a qualquer custo. Todavia, Gross (1990, cit. in O'Donohue et al., 2003) não usa o termo retórica no sentido pejorativo. O que ele pretende mostrar é que os métodos "válidos" e "racionais" da ciência são justificados, mas que também eles são altamente persuasivos. O fenômeno da expansão da psicologia cognitiva teria se beneficiado desse expediente. 
Como conclusão de seu extenso artigo, O'Donohue et al. (2003) argumentam veementemente contra a idéia de que houve uma revolução e acreditam que muitos pesquisadores foram persuadidos de que o programa cognitivista era mais poderoso e promissor, resultando num verdadeiro fenômeno de massa entre os psicólogos.

Diante do exposto até aqui, é possível afirmar que a noção de revolução cognitiva é uma obra de retórica sofisticada, uma obra de ficção que, de tanto ser repetida, passou a constituir uma espécie de "falsa memória".

\section{Implicações epistemológicas}

Por que Kuhn fez tanto sucesso, é uma questão em aberto, mas o zeitgeist da década de 60 foi bastante favorável para as revoluções. A moda, então, falou mais alto.

As razões pelas quais os psicólogos fazem o uso indevido dos conceitos da filosofia da ciência de Kuhn parecem exemplificar um tipo de processamento automático, desatento, em razão, talvez, do desconhecimento da obra de Kuhn. É, no mínimo, paradoxal justificar o status científico da psicologia utilizando um autor que explicitamente apresenta ideias que falseiam a psicologia como ciência (Leahey, 1992). Por que a Psicologia precisa obedecer aos cânones da teoria de Kuhn? É como se para ser científico o requisito é ter paradigma.

A co-existência de diferentes escolas de pensamento na psicologia é um fato notório. As diferentes psicologias continuam a existir e seus seguidores dividem "grants", espaços para publicação e congressos. A unificação da psicologia é um sonho fictício. É um mito o uso da filosofia kuhniana na sustentação científica da psicologia: é necessário haver um paradigma, depois esse paradigma torna-se anômalo e é substituído por outro, ou seja, esse é o processo revolucionário. Não há paradigma; por conseguinte, não pode haver revolução! A pulverização de escolas psicológicas trabalha contra esse objetivo: há um verdadeiro mar de teorias, modelos, métodos.

Fazendo um balanço crítico, fundado em dados históricos e bibliométricos, é possível concluir que as três grandes escolas da psicologia continuam vivas: o behaviorismo, a psicologia cognitiva e a psicanálise (Friman et al., 1993). Por exemplo, Schlinger (2008) apela para Mark Twain, afirmando que as mortes do Verbal Behavior e do Behaviorismo tem sido muito exageradas. Na outra direção, Proctor e Vu (2006) fazem uma exposição histórica da revolução cognitiva aos 50 anos, mostrando a utilidade atual da abordagem do processamento de informação, embora haja dados que mostram que essa abordagem tem muitos pontos fracos, ensejando o aparecimento de outras posições teóricas (Lopes, Lopes, \& Teixeira, 2004). 
Para finalizar, cabe a pergunta: Se a filosofia de Kuhn não sustenta o projeto científico da Psicologia, o que fazer? Por que precisamos de paradigmas e revoluções?

A resposta demandaria várias outras páginas, mas em vez de se preocupar com rótulos epistemológicos, a psicologia poderia seguir o conselho de Leahey (1992) que afirma que em lugar de uma história de revoluções, alguém poderia contar a estória da psicologia como uma narrativa das tradições de pesquisa. Nessa direção, a adoção do naturalismo normativo de Laudan (1996), uma proposta de Capaldi e Proctor (2000), surge como uma luz no fim do túnel. Não sendo nem positivismo nem relativismo, propõe estratégias interessantes para a pesquisa e a metodologia no campo da psicologia. Suas três suposições mais importantes são: uma teoria progressiva é aquela que tem mais capacidade de resolução de problemas empíricos e conceituais; a preferência de uma teoria sobre a outra se dá com base no progresso; os métodos devem ser avaliados de acordo com os mesmos critérios de avaliação das proposições empíricas.

Epistemologias dessa natureza são profiláticas e evitam a colocação das psicologias no seio de discussões políticas, mais que científicas. É preciso dotar as psicologias de capacidade de solução de problemas teóricos e metodológicos, de poder explanatório e valor preditivo. Sua multiplicidade pode ser respeitada e nenhum apelo a paradigmas e revoluções servirá de panacéia artificial para resolver seus problemas internos.

\section{Referências}

Anderson, J.R. (1995). Cognitive psychology and its implications. New York: Freeman.

Atkinson, R.C., \& Shiffrin, R.M. (1968). Human memory: a proposed system and its control processes. In K.W. Spence \& J.T. Spence (Eds.), The psychology of learning and motivation: Advances in research and theory (pp. 89-195). New York: Academic Press.

Baars, B.J. (1986). The cognitive revolution in psychology. New York: Guilford Press.

Brehm, S. S. (2008). Looking ahead: The future of psychology and APA. American Psychologist, 63, 337-344.

Broadbent. D. (1958). Perception and communication. Oxford: Pergamon.

Capaldi, E.J., \& Proctor, R.W. (2000). Laudan's normative naturalism: A useful philosophy of science for psychology. American Journal of Psychology, 113, 430-454.

Carone, I. (1998). A questão dos paradigmas na psicologia - Um balanço crítico. Psicologia em Estudo, 3, 191-200.

Carone, I. (2003). A psicologia tem paradigmas? São Paulo: Casa do Psicólogo.

Castelo Branco, C. (2007). Os militares no poder: De 1964 ao Al-5. Rio de Janeiro: Nova Fronteira.

Chomsky, N. (1959). Review of B.F. Skinner's Verbal Behavior. Language, 35, 26-58.

Crane, T. (1995). The mechanical mind: A philosophical introduction to minds, machines and mental representation. London: Penguin Books. 
Cronbach, L.J. (1957). The two disciplines of scientific psychology. American Psychologist, 12, 671-684.

De Jaager, J.J. (1970). Origins of psychometry. In J. Brozek and M.S. Sibinga (Eds.). Reaction time and mental processes. Nieuwkoop: De Graaf.

Donders, F.C. (1969). On the speed of mental processes. (Transl. By W.G. Koster). Acta Psychologica, 30, 412-431. (Trabalho original publicado em 1868).

Driver-Linn, E. (2003). Where is psychology going? Structural fault lines revealed by psychologists' use of Kuhn. American Psychologist, 58, 269-278.

Ebbinghaus, H. (1964). Memory: A contribution to experimental psychology. New York: Dove. (original publicado em 1885).

Eysenck, M.W., \& Keane, M.T. (1994). Psicologia cognitiva: Um manual introdutório. Porto Alegre: Artes Médicas.

Feyrabend, P. (1975). Against method. London: Verson.

French, C.C., \& Colman, A . M. (Eds.) (1995). Cognitive psychology. New York: Longman Publ. Inc.

Friman, P.C., Allen, K.D., Kerwin, M.L.E., \& Larzelere, R. (1993). Changes in modern psychology: A citation analysis of the Kuhnian displacement thesis. American Psychologist, 48, 658-664.

Galera, C., \& Lopes, E.J. (1995). Cronometria de processos mentais. Temas em Psicologia, 3, 1-10.

Gardner, H. (1995). A nova ciência da mente: Uma história da revolução cognitiva. São Paulo: Edusp.

Goldstein, E.B. (2005). Cognitive psychology: connecting mind, research, and everyday experience. Belmont, CA: Thomson Wadsworth.

Hassebrock, F. (1990). Tracing the cognitive revolution through a literature search. Teaching of Psychology, 17, 251-252.

Hatfield, G. (2002). Psychology, philosophy, and cognitive science: Reflections on the history and philosophy of experimental psychology. Mind \& Language, 17, 207-232.

Heidbreder, E. (1981). Psicologias do século XX. São Paulo: Mestre Jou. (Trabalho original publicado em 1933).

Hunt, H. T. (2005). Why psychology is/is not traditional science: The self-referential bases of psychological research and theory. Review of General Psychology, 9, 358-374.

James, W. (1950). Principles of psychology. New York: Dover. (Trabalho original publicado em 1890).

Johnson, D.M., \& Erneling, C.E. (1997). The future of the cognitive revolution. New York: Oxford University Press.

Kausler, D.H. (1974). Psychology of verbal learning and memory. New York: Academic Press.

Kendler, H.H. (1987). Historical foundations of modern psychology. Cal.: Brooks Cole.

Kessel, F.S., \& Bevan, W. (1985). Notes toward a history of cognitive psychology. In C.E. Buxton (Ed.) Points of view in the modern history of psychology (pp. 259-294). New York: Academic Press.

Kuhn, T.S. (1978) A estrutura das revoluções cientificas ( $2^{a}$. Ed.). São Paulo: Perspectiva. (Trabalho original publicado em 1962)

La Berge, D. (1990). Attention. Psychological Science, 1, 156-162.

Lachman, R., Lachman, J.L., \& Butterfield, E.C. (1979). Cognitive psychology and information processing: An introduction. Hillsdale, NJ.: Lawrence Erlbaum Ass.

Lakatos, I. (1981). History of science and its rational reconstructions. In I. Hacking (Ed.) Scientific revolutions (pp. 107-127). Oxford: Oxford University Press. 
Laudan, L. (1977). Progress and its problems: Towards a theory of scientific growth. Berkeley: University of California Press.

Laudan, L. (1996). Beyond positivism and relativism: Theory, method, and evidence. Bolder, CO: Westview.

Leahey, T. H. (1992). The mythical revolutions of American psychology. American Psychologist, 47, 308-318.

Levitin, D.J. (Ed.) (2002). Foundations of cognitive psychology: core readings. Cambridge, MA: MIT Press.

Lopes, E.J., \& Galera, C. (1998). A seleção de estímulos relevantes na busca visual: Processos automáticos ou controlados? Temas em Psicologia da SBP, 3, 191-198.

Lopes, E. J., Lopes, R. F.F., \& Rodrigues, C.M.L. (2000). Perspectivas do estudo da mente: da psicologia cognitiva às neurociências. Revista da Sociedade de Psicologia do Triângulo Mineiro, 3, 6-11.

Lopes, E.J., Lopes, R.F.F., \& Teixeira, J.F. (2004). A psicologia cognitiva experimental cinqüenta anos depois: A crise do paradigma do processamento de informação. Paidéia Cadernos de Psicologia e Educação, FFCLRP-USP, 14, 17-26.

Mandler, G. (1985). Cognitive psychology: An essay in cognitive science. Hillsdale, NJ: Lawrence Erlbaum.

Masterman, M. (1979). A natureza de um paradigma. In I. Lakatos \& A . Musgrave (Orgs.). A critica e o desenvolvimento do conhecimento. São Paulo: Cultrix e Edusp.

Matlin, M. (2004). Psicologia cognitiva (5. ${ }^{a}$ Ed.). Rio de Janeiro: LTC.

Miller, G. A . (1956). The magical number seven, plus or minus two: Some limits on our capacity for processing information. Psychological Review, 63, 81-97.

Miller, G. A. (2003). The cognitive revolution: a historical perspective. Trends in cognitive sciences, 7, 141-144.

Neisser, U. (1967). Cognitive psychology. New York: Appleton-Century-Crofts.

O' Donohue, W., Ferguson, K.E., \& Naugle, A. E. (2003). The structure of the cognitive revolution: An examination from the philosophy of science. The Behavior Analyst, 26, 85-110.

Popper, K. (1999). A lógica da pesquisa científica. São Paulo: Cultrix. (Trabalho original publicado em 1959).

Popper, K. (1979). A ciência normal e seus perigos. In I. Lakatos \& A . Musgrave (Orgs.). A crítica e o desenvolvimento do conhecimento. São Paulo: Cultrix e Edusp.

Posner, M.I., \& Levitin, D.J. (1999). Imaging the future. In R.L. Solso (Ed.), Mind and brain sciences in the 21 st. Century. Cambridge, Mass: MIT Press.

Proctor, R.W., \& Vu, K.P.L. (2006). The cognitive revolution at age 50: Has the promise of the human information-processing approach been fulfilled? International Journal of Human-Computer Interaction, 21, 253-284.

Robins, R.W., Gosling, S.D., \& Craik, K.H. (1999). An empirical analysis of trends in psychology. American Psychologist, 54, 117-128.

Schlinger, H. D. (2008). The long good-bye: Why B.F. Skinner's Verbal Behavior is alive and well on the $5 \mathrm{O}^{\mathrm{TH}}$ anniversary of its publication. The Psychological Record, 58, 329-337.

Shannon, C.E., \& Weaver, W. (1949). The mathematical theory of communication. Urbana, IL: University of Illinois Press.

Skinner, B.F. (1978). O comportamento verbal. São Paulo: Cultrix/Edusp. (Trabalho original publicado em 1957).

Skinner, B.F. (1991). Questões recentes na análise comportamental. Campinas: Papirus. 
Solso, R. L. (1999). Mind and brain sciences in the 21th century. Cambridge, Mass.: MIT Press. Spear, J.H. (2007). Prominent schools or other active specialties? A fresh look at some trends in psychology. Review of General Psychology, 11, 363-380.

Sternberg, R.J. (2007). Psicologia cognitiva. (4. ${ }^{\text {a }}$ Ed.). Porto Alegre: Artes Médicas.

Teixeira, J.F.(1998). Mentes e máquinas: Uma introdução à ciência cognitiva. Porto Alegre: Artes Médicas Sul.

Teixeira, J.F. (2000). Mente, cérebro e cognição. Petrópolis: Vozes.

Thagard, P. (1998). Mente: Introdução à ciência cognitiva. Porto Alegre: ArtMed.

Thompson, R.F. (1999). Will the mind become the brain in the $21^{\text {st }}$. century? In R.L. Solso (Ed.), Mind and brain sciences in the 21 st. Century. Cambridge, Mass: MIT Press.

Tolman, E.C. (1932). Purposive behaviour in animals and men. New York: Appleton-Century-Crofts.

\section{A Critical Analysis of the Cognitive Revolution: pistemological Implications}

This paper presents a critical review of the cognitive revolution in psychology and the epistemological implications for the scientific status of psychology. Cognitive psychology was born in the fifties and the information processing is its main "paradigm". It has been diffused as a scientific revolution as proposed by T. Kuhn. This proposal is false and raises doubts about psychology as a science. I suggest the abandonment of the Kuhn's philosophy on account of the normative naturalism, a useful solution for psychologies.

KEYWORDS: Cognitive Psychology; Paradigm; Scientific Revolution; Cognitive Revolution; Normative Naturalism. 